\title{
Evaluation of Fuzzy Quantified Sentences: Keeping the Boolean Properties
}

\author{
D. Sánchez \\ European Centre for Soft Computing \\ 33600 Mieres, Asturias, Spain \\ Email: daniel.sanchezf@softcomputing.es \\ Dept. Computer Science and A.I. \\ University of Granada, 18071 Granada, Spain \\ Email: daniel@decsai.ugr.es
}

\author{
M. Delgado, M.A. Vila, J. Chamorro-Martínez \\ Dept. Computer Science and A.I. \\ University of Granada, 18071 Granada, Spain \\ Email: mdelgado@ugr.es, \{vila,jesus\}@decsai.ugr.es
}

\begin{abstract}
In this paper we describe our approach to fuzzy quantification using an alternative representation of fuzziness recently proposed by the authors. We show the approach both in the evaluation of quantified sentences and calculations with linguistic restrictions in the form of quantifiers.
\end{abstract}

\section{INTRODUCTION}

In the framework of Computing with Words and Perceptions [1], an important issue is the evaluation of the accomplishment degree of linguistic expressions involving quantifiers, called quantified sentences. Zadeh's framework for quantification [2], considers expressions of the form " $Q$ of $X$ are $A$ " or " $Q$ of $D$ are A", called type I and type II sentences, respectively. Here, $\mathrm{Q}$ is a linguistic quantifier, $\mathrm{X}$ is a (finite) crisp set, and A,D are fuzzy subsets of $\mathrm{X}$. There are many different approaches for evaluating the accomplishment degree of this type of sentences, see among others [2], [3], [4], [5], [6], [7], [8], [9], [10], [11].

Several authors have proposed properties that quantification methods should verify in order to obtain intuitive results from the human point of view. For instance, the property called "Internal negation": the evaluation of "Q of A are D" must be equal to the evaluation of "antQ of A are $\neg \mathrm{D}$ ", where antQ is the antonym of $\mathrm{Q}$ defined as $\operatorname{ant} \mathrm{Q}(\mathrm{x})=\mathrm{Q}(1-\mathrm{x})$, or the property of "Duality": the evaluation of "Q of A are D" is equal to the evaluation of " $(\neg$ antQ) of A are $\neg$ D". Some of these intuitive properties are those of Boolean algebras, and their fulfilment depends not only on the evaluation method, but on the operations employed on fuzzy sets.

Standar Fuzzy Set Theories (FST) are defined by triples $(T, S, N)$ where $T$ is a t-norm, $S$ a t-conorm, and $N$ a fuzzy negation. It is well known that for every basic property of Boolean algebras, there is at least one FST that satisfy it, but there are some couples of properties that cannot be satisfied at the same time; for example, Dubois and Prade showed in [12], [13] that no standard FST can satisfy idempotency (as well as mutual distributivity) together with the laws of excluded middle and non-contradiction. Hence, no standard FST satisfy all the basic properties of Boolean algebras.

As a consequence there are intuitive properties that cannot be satisfied by the evaluation of fuzzy quantified sentences. For instance, consider the following sentences:

- All $A$ are $A \wedge A$

- All $X$ are $A \vee \neg A$

- No $X$ is $A \wedge \neg A$

where $\wedge, \vee$, and $\neg$ are to be calculated by means of an FST, the quantifier $A l l$ is defined on $[0,1]$ as $\operatorname{All}(\alpha)=1$ iff $\alpha=1$, and 0 otherwise, and the quantifier $N o$ can be defined as the negation of the quantifier Exists as $N o(\alpha)=1$ iff $\alpha=0$, and 0 otherwise. Intuitively, the expected accomplishment degree of these sentences is 1 , but this can be accomplished for all the sentences simultaneously iff for any fuzzy cardinality card:

- $\operatorname{card}(A \wedge A) / \operatorname{card}(A)=1$, which implies $A \wedge A=A$ (idempotency),

- $\operatorname{card}(A \vee \neg A) / \operatorname{card}(X)=1$, which implies $X=A \vee \neg A$ (excluded middle), and

- $\operatorname{card}(A \wedge \neg A) / \operatorname{card}(X)=0$, which implies $A \wedge \neg A=\emptyset$ (non-contradiction).

However, this is not possible since as we mentioned before, no FST can satisfy these three properties at the same time.

The fact that no FST satisfies all the Boolean properties is usually perceived as positive. For instance, the fact that $A \wedge \neg A \neq \emptyset$ is usually justified since it allows elements to be at the same time in the representation of a property and its negation, something that is characteristic of fuzzy concepts. The question then is, do we have to choose between having a suitable representation of fuzzy concepts and keeping all the Boolean properties when they are intuitively convenient, like in the case of quantification?

The answer is no. In [14] we have proposed a representation of fuzzy concepts called Representation by Levels (RL), akin to the notions of gradual element and gradual sets introduced by Dubois and Prade [15], [16], that allows elements to verify a concept and its negation at the same time that all Boolean properties are kept. In this approach, fuzzy concepts are represented by an assignment of crisp sets to levels of fulfilment of a property. RLs are not fuzzy sets in general, though the latter can be seen as a particular case of the former. In addition, RLs corresponding to fuzzy sets are not closed under logical operations of conjunction, disjunction and 
negation. Hence, the whole proposal is not an alternative to standard FST for operating with fuzzy sets, but an alternative to the representation of fuzzy concepts, having a Boolean structure. However, fuzzy sets are very important in our approach, since they are a natural intermediate step between humans and RLs. In particular, humans provide RLs as fuzzy sets.

The paper is organized as follows: we introduce the representation by levels in section II. Our approach to the evaluation of quantified sentences using RLs is described in section III. An approach to solving an interesting kind of question in Computing with Words based on RLs is provided in section IV. Section V contains our conclusions and future research work.

\section{REPRESENTATION BY LEVELS}

We propose to represent fuzzy concepts defined on a set of objects $X$ by means of an assignment to values in $[0,1]$ of crisp sets which are not necessarily nested. This non-nestedness is the main difference of our approach with respect to the usual representation of fuzzy sets by means of $\alpha$-cuts. Of course, the latter is a particular case of the former and hence, from the representation point of view, fuzzy sets are particular cases of RLs. Another important difference is that we consider a finite set of levels (human beings are not able to distinguish an infinite amount of levels). Finiteness is not a problem in practice, since we deal with finite computers and a finite set of objects. The interpretation of levels is that of possible degrees of relaxation of the concept. The level 1 corresponds to the most restrictive, strict view of the concept, and the corresponding representation contains those objects for which there is no doubt that they satisfy the concept. On the other extreme, the value 0 means no restriction at all, i.e., at this level every object satisfy every atomic concept. Hence, this level is never employed in the representation of concepts. The semantics of intermediate levels is based on the distance to 1 and 0 , i.e., level 0.5 is halfway between being totally strict and no strict at all.

We assume that for every concept there is a finite set of levels $\Lambda=\left\{\alpha_{1}, \ldots, \alpha_{m}\right\}$ satisfying $1=\alpha_{1}>\alpha_{2}>\cdots>$ $\alpha_{m}>\alpha_{m+1}=0, m \geq 1$. We define a representation by levels of a fuzzy concept on $X$ as follows:

Definition 1. An RL is a pair $(\Lambda, \rho)$ where $\Lambda$ is a set of levels and $\rho$ is a function

$$
\rho: \Lambda \rightarrow \mathcal{P}(X)
$$

We shall denote a concept and its corresponding representation in the same way, so that $A$ is a concept that will be represented by an RL $A=\left(\Lambda_{A}, \rho_{A}\right)$.

Definition 2. The set of crisp representatives $\Omega_{A}$ of an RL $\left(\Lambda_{A}, \rho_{A}\right)$ is

$$
\Omega_{A}=\left\{\rho_{A}(\alpha) \mid \alpha \in \Lambda_{A}\right\}
$$

The information represented by an RL $A$ is not restricted to the levels in $\Lambda_{A}$, but can conveniently be extended to any other level in $\alpha \in(0,1]$ as follows:
Definition 3. Let $(\Lambda, \rho)$ be an RL with $\Lambda=\left\{\alpha_{1}, \ldots, \alpha_{m}\right\}$ satisfying $1=\alpha_{1}>\alpha_{2}>\cdots>\alpha_{m}>\alpha_{m+1}=0$. Let $\alpha \in(0,1]$ and $\alpha_{i}, \alpha_{i+1} \in \Lambda$ such that $\alpha_{i} \geq \alpha>\alpha_{i+1}$. Then

$$
\rho(\alpha)=\rho\left(\alpha_{i}\right)
$$

Definition 4. Let $(\Lambda, \rho)$ be an RL. We define the associated probability distribution $\omega: \Lambda \rightarrow[0,1]$ as

$$
\omega\left(\alpha_{i}\right)=\alpha_{i}-\alpha_{i+1} .
$$

$\omega(\alpha)$ represents the amount of evidence assigned to level $\alpha$. On this basis, a probability assignment $m: \Omega \rightarrow[0,1]$ can be associated to the crisp representatives of an RL in the usual way:

$$
m(Y)=\sum_{\alpha_{i} \mid Y=\rho\left(\alpha_{i}\right)} \alpha_{i}-\alpha_{i+1} \quad \forall Y \in \Omega .
$$

Notice that the set $\Omega$ endowed with $m$ is a random set [15], [16]. It is easy to see that different RLs may yield the same random set. This is the case for example with the RLs $A=\{(1,\{a, b\}),(0.5,\{c, d\})\}$ and $B=$ $\{(1,\{c, d\}),(0.5,\{a, b\})\}$; in both cases, the random set obtained is given by $m(\{a, b\})=m(\{c, d\})=0.5$. We say that the random set defined by a function $m_{A}$ obtained from an RL $A$ is a summary of the information in $A$, since it represents the evidence associated to crisp representatives of the RL $A$ by losing the information about the indexing structure.

Using this random set, it is possible to obtain an objectcentered summary of the information given by an RL $A$ as a fuzzy set by means of the fixed (or single) point coverage function of the corresponding random set $m_{A}$ as proposed by Dubois and Prade [17] (corresponding to a contour function in the sense of Shafer [18]). The associated fuzzy summary $\nu_{A}: X \rightarrow[0,1]$ is

$$
\nu_{A}(x)=\sum_{Y \in \Omega_{A} \mid x \in Y} m_{A}(Y)
$$

Since the same fuzzy set can be obtained from many different random sets [19], [20], we are again summarizing in the sense of losing part of the information represented by the random set. What remains is the evidence that the RL contains each individual object.

This summary is consistent with the representation of fuzzy sets by means of RLs, since for a fuzzy set $A$ represented by an $\mathrm{RL}\left(\Lambda_{A}, \rho_{A}\right)$ it is well known that $\nu_{A}(x)=A(x)$. However, as we shall see, this is not always the case with the RLs obtained after logical operations, even when operating on fuzzy sets.

The idea of fuzzy summary of an RL is important when the information given by the representation is to be provided to a human user, since fuzzy sets are much easier to understand and to assess than RLs.

\section{A. Operations}

Operations with RLs rely on the following ideas:

1) The meaning of a level is the same when applied to the representation of any fuzzy concept. This is a generalization of the notion of single membership scale 
in fuzzy sets (commensurability assumption), i.e., let $F$ and $G$ be fuzzy subsets of $X$, then $F(x)=G\left(x^{\prime}\right)$ means that $x$ is $F$ just like $x^{\prime}$ is $G$. In our case, we consider that it makes sense to see the representation of a knowledge base $\mathrm{KB}$ at a certain level as a knowledge base consisting of the representation at that level of all the concepts that form KB.

2) Crisp operations are extended to RLs by operating at each level independently. In general, let $f$ : $\mathcal{P}(X)^{n} \rightarrow \mathcal{P}(X)$ be a crisp operation. Then, $f$ is extended to RLs as follows: let $\left(P_{1}, \ldots, P_{n}\right)$ be fuzzy concepts defined on $X$ with $P_{i}$ represented by an $\mathrm{RL}\left(\Lambda_{P_{i}}, \rho_{P_{i}}\right)$. Then, $f\left(P_{1}, \ldots, P_{n}\right)$ is represented by $\left(\Lambda_{f\left(P_{1}, \ldots, P_{n}\right)}, \rho_{f\left(P_{1}, \ldots, P_{n}\right)}\right)$ where

$$
\Lambda_{f\left(P_{1}, \ldots, P_{n}\right)}=\bigcup_{1 \leq i \leq n} \Lambda_{P_{i}}
$$

and, $\forall \alpha \in \Lambda_{f\left(P_{1}, \ldots, P_{n}\right)}$,

$$
\rho_{f\left(P_{1}, \ldots, P_{n}\right)}(\alpha)=f\left(\rho_{P_{1}}(\alpha), \ldots, \rho_{P_{n}}(\alpha)\right)
$$

Definition 5. Let $P, Q$ be fuzzy concepts represented by $\left(\Lambda_{P}, \rho_{P}\right),\left(\Lambda_{Q}, \rho_{Q}\right)$. Then, $P \wedge Q$ and $P \vee Q$ are fuzzy concepts represented by $\left(\Lambda_{P \wedge Q}, \rho_{P \wedge Q}\right)$ and $\left(\Lambda_{P \vee Q}, \rho_{P \vee Q}\right)$, respectively, where

$$
\Lambda_{P \wedge Q}=\Lambda_{P \vee Q}=\Lambda_{P} \cup \Lambda_{Q}
$$

with, $\forall \alpha \in(0,1]$,

$$
\rho_{P \wedge Q}(\alpha)=\rho_{P}(\alpha) \cap \rho_{Q}(\alpha)
$$

and

$$
\rho_{P \vee Q}(\alpha)=\rho_{P}(\alpha) \cup \rho_{Q}(\alpha)
$$

Definition 6. Let $P$ be a fuzzy concept represented by $\left(\Lambda_{P}, \rho_{P}\right)$. Then, $\neg P$ is a fuzzy concept represented by $\left(\Lambda_{\neg P}, \rho_{\neg P}\right)$, where

$$
\Lambda_{\neg P}=\Lambda_{P}
$$

and, $\forall \alpha \in(0,1]$,

$$
\rho_{\neg P}(\alpha)=\overline{\rho_{P}(\alpha)}
$$

where $\bar{Y}$ is the usual set complement of a crisp set $Y$.

As an example of negation, consider an universe $X=$ $\left\{x_{1}, \ldots, x_{5}\right\}$ and the fuzzy set

$$
A=1 / x_{1}+0.8 / x_{2}+0.5 / x_{3}+0.4 / x_{5}
$$

Table I shows the RL-representations for $A$ and $\neg A$ on the level-set

$$
\Lambda_{A}=\Lambda_{\neg A}=\{1,0.8,0.5,0.4\} .
$$

As we can see, the representation of the negation of a fuzzy set (e.g. $\neg A$ ) is not the set of $\alpha$-cuts of the complement of the corresponding fuzzy set (e.g. the fuzzy set $\bar{A}(x)=1-$ $A(x)$ ). In fact, the representations of $\neg A$ and $\neg B$ are not fuzzy sets, and this result holds for any non-crisp fuzzy set. Notice however that the result makes sense since if we assume that the set of objects that satisfy the concept $A$ at a certain level $\alpha$ is $\rho_{A}(\alpha)$, then it is perfectly reasonable to assume that the

\begin{tabular}{|c|l|l|}
\hline$\alpha$ & $\rho_{A}(\alpha)$ & $\rho_{\neg A}(\alpha)$ \\
\hline 1 & $\left\{x_{1}\right\}$ & $\left\{x_{2}, x_{3}, x_{4}, x_{5}\right\}$ \\
\hline 0.8 & $\left\{x_{1}, x_{2}\right\}$ & $\left\{x_{3}, x_{4}, x_{5}\right\}$ \\
\hline 0.5 & $\left\{x_{1}, x_{2}, x_{3}\right\}$ & $\left\{x_{4}, x_{5}\right\}$ \\
\hline 0.4 & $\left\{x_{1}, x_{2}, x_{3}, x_{5}\right\}$ & $\left\{x_{4}\right\}$ \\
\hline
\end{tabular}

TABLE I

NEgATION OF $A$.

set of objects that do not satisfy $A$ at the same level is $\overline{\rho_{A}(\alpha)}$. In addition, the following proposition holds:

Proposition 1. Let $A$ be a fuzzy set represented by an RL. Then, $\forall x \in X$

$$
\nu_{\neg A}(x)=\bar{A}(x)=1-A(x)
$$

Proof: Let $x \in X$ with degree $A(x)$. Then,

$$
\sum_{Y \in \Omega_{A} \mid x \in Y} m_{A}(Y)=A(x)
$$

Since $\sum_{Z \in \Omega_{\neg A}} m_{\neg A}(Z)=1$ and $x \in Y \in \Omega_{A}$ implies $x \notin$ $\bar{Y} \in \Omega_{A}$, and $m_{A}(Y)=m_{\neg A}(\bar{Y})$, we have

$$
\sum_{\bar{Y} \in \Omega_{\neg A} \mid x \in \bar{Y}} m_{\neg A}(\bar{Y})=1-A(x)
$$

As an example of combination of operations, Tables II and III show the representation of several concepts derived from $A$ and $B$. In particular, in Table II we have an example of $\mathrm{RL}$, that of the concept $A \wedge \neg B$, in which the different crisp realizations are not nested, showing that RLs other than those corresponding to fuzzy sets and the complement of fuzzy sets are possible, and hence RLs without any particular relationship between the crisp realizations at different levels may arise naturally by performing intersection, union, and complement of RLs only, even if the original RLs correspond to fuzzy sets.

\section{B. Properties}

It is obvious that RLs satisfy the Classical Preservation Principle [21], i.e., crisp concepts can be represented by means of RLs, the representatives being the same at every level, and in that case the crisp operations are the same, and all the properties are kept. This is the basic property that any FST and fuzziness representation model must satisfy. However, there is a far more important and strong result about operations with RLs:

Proposition 2. Operations on RLs satisfy all the properties of Boolean logic.

Proof: Immediate since the logical operations between RLs are performed via the corresponding set operations on crisp sets at each level, that satisfy the corresponding properties of Boolean logic, and the equivalence of RLs is defined in terms of equality of the representatives at every level.

By proposition 2, basic properties of Boolean logic that cannot be satisfied simultaneously by any standard FST, as well as those derived properties of Boolean logic that cannot 


\begin{tabular}{|c|l|l|l|l|l|l|}
\hline$\alpha$ & $\rho_{A}(\alpha)$ & $\rho_{\neg A}(\alpha)$ & $\rho_{B}(\alpha)$ & $\rho_{\neg B}(\alpha)$ & $\rho_{A \wedge \neg B}(\alpha)$ & $\rho_{B \wedge \neg A}(\alpha)$ \\
\hline 1 & $\left\{x_{1}\right\}$ & $\left\{x_{2}, x_{3}, x_{4}, x_{5}\right\}$ & $\emptyset$ & $X$ & $\left\{x_{1}\right\}$ & $\emptyset$ \\
\hline 0.9 & $\left\{x_{1}\right\}$ & $\left\{x_{2}, x_{3}, x_{4}, x_{5}\right\}$ & $\left\{x_{1}\right\}$ & $\left\{x_{2}, x_{3}, x_{4}, x_{5}\right\}$ & $\emptyset$ & $\emptyset$ \\
\hline 0.8 & $\left\{x_{1}, x_{2}\right\}$ & $\left\{x_{3}, x_{4}, x_{5}\right\}$ & $\left\{x_{1}\right\}$ & $\left\{x_{2}, x_{3}, x_{4}, x_{5}\right\}$ & $\left\{x_{2}\right\}$ & $\emptyset$ \\
\hline 0.6 & $\left\{x_{1}, x_{2}, x_{3}\right\}$ & $\left\{x_{4}, x_{5}\right\}$ & $\left\{x_{1}, x_{3}\right\}$ & $\left\{x_{2}, x_{4}, x_{5}\right\}$ & $\left\{x_{2}\right\}$ & $\emptyset$ \\
\hline 0.5 & $\left\{x_{1}, x_{2}, x_{3}\right\}$ & $\left\{x_{4}, x_{5}\right\}$ & $\left\{x_{1}, x_{3}, x_{4}\right\}$ & $\left\{x_{2}, x_{5}\right\}$ & $\left\{x_{2}\right\}$ & $\left\{x_{4}\right\}$ \\
\hline 0.4 & $\left\{x_{1}, x_{2}, x_{3}, x_{5}\right\}$ & $\left\{x_{4}\right\}$ & $\left\{x_{1}, x_{3}, x_{4}\right\}$ & $\left\{x_{2}, x_{5}\right\}$ & $\left\{x_{2}, x_{5}\right\}$ & $\left\{x_{4}\right\}$ \\
\hline
\end{tabular}

TABLE II

SEVERAL CONCEPTS DERIVED FROM THE FUZZY SETS $A$ AND $B$ (I).

\begin{tabular}{|c|l|l|l|l|l|}
\hline$\alpha$ & $\rho_{A \wedge B}(\alpha)$ & $\rho_{A \vee B}(\alpha)$ & $\rho_{\neg(A \wedge B)}(\alpha)$ & $\rho_{\neg(A \vee B)}(\alpha)$ & $\rho_{\neg A \vee \neg B}(\alpha)$ \\
\hline 1 & $\emptyset$ & $\left\{x_{1}\right\}$ & $X$ & $\left\{x_{2}, x_{3}, x_{4}, x_{5}\right\}$ & $X$ \\
\hline 0.9 & $\left\{x_{1}\right\}$ & $\left\{x_{1}\right\}$ & $\left\{x_{2}, x_{3}, x_{4}, x_{5}\right\}$ & $\left\{x_{2}, x_{3}, x_{4}, x_{5}\right\}$ & $\left\{x_{2}, x_{3}, x_{4}, x_{5}\right\}$ \\
\hline 0.8 & $\left\{x_{1}\right\}$ & $\left\{x_{1}, x_{2}\right\}$ & $\left\{x_{2}, x_{3}, x_{4}, x_{5}\right\}$ & $\left\{x_{3}, x_{4}, x_{5}\right\}$ & $\left\{x_{2}, x_{3}, x_{4}, x_{5}\right\}$ \\
\hline 0.6 & $\left\{x_{1}, x_{3}\right\}$ & $\left\{x_{1}, x_{2}, x_{3}\right\}$ & $\left\{x_{2}, x_{4}, x_{5}\right\}$ & $\left\{x_{4}, x_{5}\right\}$ & $\left\{x_{2}, x_{4}, x_{5}\right\}$ \\
\hline 0.5 & $\left\{x_{1}, x_{3}\right\}$ & $\left\{x_{1}, x_{2}, x_{3}, x_{4}\right\}$ & $\left\{x_{2}, x_{4}, x_{5}\right\}$ & $\left\{x_{5}\right\}$ & $\left\{x_{2}, x_{4}, x_{5}\right\}$ \\
\hline 0.4 & $\left\{x_{1}, x_{3}\right\}$ & $X$ & $\left\{x_{2}, x_{4}, x_{5}\right\}$ & $\emptyset$ & $\left\{x_{2}, x_{4}, x_{5}\right\}$ \\
\hline
\end{tabular}

TABLE III

SEVERAL CONCEPTS DERIVED FROM CONCEPTS $A$ AND $B$ (II).

be satisfied by any standard FST, hold simultaneously for RLs. In particular, idempotency and law of excluded middle and non-contradiction hold.

Let us highlight that at the same time that these properties hold, the important feature of fuzzy sets that an element may appear both in the representation of a concept and its negation is also kept. As an example, the element $x_{2}$ appears in the level representation of both concepts $A$ and $\neg A$ (see Table II). The only restriction is that an element cannot appear in the representation of both concepts at the same level. This way, levelwise operations guarantee the excluded middle property at the cost of truth-functionality.

\section{EVAlUation OF QUANTIFIED SENTENCES}

We shall consider the evaluation of quantified sentences of type II since type I sentences are a particular case of type II sentences, under the following assumptions:

- Q is a fuzzy quantifier

- A,D are imprecise properties defined on a finite, crisp set $\mathrm{X}$ by RL-representations $\left(\Lambda_{A}, \rho_{A}\right)$ and $\left(\Lambda_{D}, \rho_{D}\right)$, respectively.

Notice that, since fuzzy sets are particular cases of RLrepresentations, the proposal in this section is also valid for the evaluation of quantified sentences when properties A,D are represented by fuzzy sets. First, in the next section we propose an evaluation by levels. Then, in section III-B, we integrate this information into a single value.

\section{A. RL-evaluation}

Definition 7. The evaluation of $\mathrm{E} \equiv$ " $\mathrm{Q}$ of $\mathrm{D}$ are $\mathrm{A}$ " is defined by $\left(\Lambda_{E}, \rho_{E}\right)$, where

$$
\Lambda_{E}=\Lambda_{A} \cup \Lambda_{D}
$$

and, $\forall \alpha \in \Lambda_{E}$,

$$
\rho_{E}(\alpha)=Q\left(\frac{\left|\rho_{A \wedge D}(\alpha)\right|}{\left|\rho_{D}(\alpha)\right|}\right)=Q\left(\frac{\left|\rho_{A}(\alpha) \cap \rho_{D}(\alpha)\right|}{\left|\rho_{D}(\alpha)\right|}\right)
$$

As an example, consider the fuzzy sets $A$ and $B$ employed in the previous section. Table IV shows a set of quantified sentences involving properties $\mathrm{A}$ and $\mathrm{D}=\mathrm{B}$, using the quantifier $Q_{m o s t}$ defined as in Equation 17. Table V shows the corresponding RL-evaluation of the sentences in table IV and the same sentences but replacing the quantifiers by $Q(x)=x\left(E_{7}\right.$ is $E_{1}$ replacing the quantifier, and so on). The latter correspond to the measure of the relative cardinality of the corresponding properties as well.

$$
Q_{\text {most }}(x)= \begin{cases}0 & x \leq 0.5 \\ 1 & x \geq 0.75 \\ 4(x-0.5) & \text { otherwise }\end{cases}
$$

From definition 7 it is obvious that RL-evaluation performs a crisp evaluation on each level, where there are crisp representatives of the imprecise properties A and D. Since both logical and arithmetic operations are performed by definition in each level independently, all the intuitive properties of quantification on crisp data are preserved.

\section{B. Numerical evaluation}

The evaluation of quantified sentences yields usually a number in $[0,1]$. We can obtain such summary of the evaluation when that is the final, expected result of our system; otherwise, following the ideas of RL-representation, we would proceed operating in each level independently.

In this paper we propose to summarize the information given by the RL-evaluation as follows:

Definition 8. The numerical summary $S(E)$ of a RLevaluation $\mathrm{E}$ defined by $\left(\Lambda_{E}, \rho_{E}\right)$ is given by

$$
S(E)=\sum_{\beta \in \Omega_{E}} m_{E}(\beta) \cdot \beta
$$

Table VI shows the evaluation of quantified sentences $E_{1}$ $E_{12}$ following definition 8 . 


\begin{tabular}{|l|l|||l|l|||l|l|}
\hline$E_{1}$ & $Q_{\text {most }}$ of A are D & $E_{3}$ & $Q_{\text {most }}$ of $\neg$ A are D & $E_{5}$ & $\exists$ of A are $\neg$ A \\
\hline$E_{2}$ & $Q_{\text {most }}$ of A are $\neg \mathrm{D}$ & $E_{4}$ & $Q_{\text {most }}$ of $\neg$ A are $\neg$ D & $E_{6}$ & $\exists$ of $(\mathrm{A} \wedge \neg$ D) are D \\
\hline
\end{tabular}

TABLE IV

SOME QUANTIFIED SENTENCES INVOLVING A AND D

\begin{tabular}{|c|l|l|l|l|l|l||l|l|l|l|l|l|}
\hline$\alpha$ & $E_{1}$ & $E_{2}$ & $E_{3}$ & $E_{4}$ & $E_{5}$ & $E_{6}$ & $E_{7}$ & $E_{8}$ & $E_{9}$ & $E_{10}$ & $E_{11}$ & $E_{12}$ \\
\hline 1 & 0 & 1 & 0 & 1 & 0 & 0 & 0 & 1 & 0 & 1 & 0 & 0 \\
\hline 0.9 & 1 & 0 & 0 & 1 & 0 & 0 & 1 & 0 & 0 & 1 & 0 & 0 \\
\hline 0.8 & 0 & 0 & 0 & 1 & 0 & 0 & $1 / 2$ & $1 / 2$ & 0 & 1 & 0 & 0 \\
\hline 0.6 & $2 / 3$ & 0 & 0 & 1 & 0 & 0 & $2 / 3$ & $1 / 3$ & 0 & 1 & 0 & 0 \\
\hline 0.5 & $2 / 3$ & 0 & 0 & 0 & 0 & 0 & $2 / 3$ & $1 / 3$ & $1 / 2$ & $1 / 2$ & 0 & 0 \\
\hline 0.4 & 0 & 0 & 1 & 0 & 0 & 0 & $1 / 2$ & $1 / 2$ & 1 & 0 & 0 & 0 \\
\hline
\end{tabular}

TABLE V

RL-EVALUATION OF SENTENCES IN TABLE IV $\left(E_{1}-E_{6}\right)$ AND THE SAME SENTENCES WITH THE QUANTIFIER $Q(x)=x\left(E_{7}-E_{12}\right)$

\begin{tabular}{|l|l|l|l|l|l||l|l|l|l|l|l|}
\hline$S\left(E_{1}\right)$ & $S\left(E_{2}\right)$ & $S\left(E_{3}\right)$ & $S\left(E_{4}\right)$ & $S\left(E_{5}\right)$ & $S\left(E_{6}\right)$ & $S\left(E_{7}\right)$ & $S\left(E_{8}\right)$ & $S\left(E_{9}\right)$ & $S\left(E_{10}\right)$ & $S\left(E_{11}\right)$ & $S\left(E_{12}\right)$ \\
\hline 0.23 & 0.1 & 0.4 & 0.5 & 0 & 0 & 0.53 & 0.46 & 0.45 & 0.55 & 0 & 0 \\
\hline
\end{tabular}

TABLE VI

NUMERICAL EVALUATION OF SENTENCES IN TABLE IV $\left(E_{1}-E_{6}\right)$ AND THE SAME SENTENCES WiTH THE QUANTIFIER $Q(x)=x\left(E_{7}-E_{12}\right)$

\begin{tabular}{|c|l|l|l|l|l|l|l|}
\hline$\alpha$ & $A=Q_{h}$ & $B=Q_{f}$ & $A \vee B$ & $Q_{m}$ & $C$ & $\neg D$ & $D$ \\
\hline 1 & {$[0.5,0.5]$} & {$[0,0.1]$} & {$[0.5,0.6]$} & {$[0.8,1]$} & {$[0.32,0.5]$} & {$[0.82,1]$} & {$[0,0.18]$} \\
\hline 0.8 & {$[0.48,0.52]$} & {$[0,0.14]$} & {$[0.48,0.66]$} & {$[0.78,1]$} & {$[0.2652,0.52]$} & {$[0.7452,1]$} & {$[0,0.2548]$} \\
\hline 0.6 & {$[0.46,0.54]$} & {$[0,0.18]$} & {$[0.46,0.72]$} & {$[0.76,1]$} & {$[0.2128,0.54]$} & {$[0.6728,1]$} & {$[0,0.3242]$} \\
\hline 0.4 & {$[0.44,0.56]$} & {$[0,0.22]$} & {$[0.44,0.78]$} & {$[0.74,1]$} & {$[0.1628,0.56]$} & {$[0.6028,1]$} & {$[0,0.3972]$} \\
\hline 0.2 & {$[0.42,0.58]$} & {$[0,0.26]$} & {$[0.42,0.84]$} & {$[0.72,1]$} & {$[0.1152,0.58]$} & {$[0.5352,1]$} & {$[0,0.4648]$} \\
\hline
\end{tabular}

TABLE VII

CALCUlating THE QUANTIFIER FOR $D$ BY LEVEls.

Let us remark again that, as the intuition suggests, the final evaluation of sentences $E_{5}, E_{6}, E_{11}$ and $E_{12}$ is 0 , though any existing method would give a greater value. This is because when representing imprecision by means of fuzzy sets, $A \cap \bar{A} \neq \emptyset$ in general. Using RL-representations, we obtain a coherent result whilst representing properly the imprecision of the properties.

\section{COMPUTING WITH WORDS USING REPRESENTATION BY LEVELS}

Let us suppose that given a set $X$ we are told that around half of the elements in $X$ satisfy $A$, a few satisfy $B \wedge \neg A$, and most of the rest, i.e., most of those that don't satisfy $A \vee$ $B$, satisfy $C$. How many elements satisfy $D=\neg A \wedge \neg B \wedge$ $\neg C$ ? This is an example of the kind of questions addressed in Computing with Words.

Assuming that specific fuzzy sets are given representing the quantifiers around half $\left(Q_{h}\right)$, a few $\left(Q_{f}\right)$, and most $\left(Q_{m}\right)$, the problem here is to try to come up with a quantifier for the amount of elements that satisfy $D$.

We will consider the RL of each quantifier. Assuming they are convex fuzzy subsets, which seems natural, the representation consists of a collection of nested subintervals of $[0,1]$. Following the ideas of RLs, we shall operate in each level independently, and then we shall integrate the whole information. It is important to remark that, in this kind of questions, we are dealing with a lack of information. That is, the quantifiers are fuzzy sets employed in a disjunctive way, since the exact amount of elements satisfying every property is not known. Quantifiers are just restrictions representing the available knowledge.

Suppose that in a certain level $\alpha$ the subintervals of $[0,1]$ corresponding to the quantifiers are $\left[a_{h}, b_{h}\right]$ for around half, $\left[a_{f}, b_{f}\right]$ for $a$ few, and $\left[a_{m}, b_{m}\right]$ for most. The restriction given in the question can then be calculated as follows: first

$$
\left[a_{A \vee B}, b_{A \vee B}\right]=\left[a_{h}+a_{f}, \min \left\{1, b_{h}+b_{f}\right\}\right]
$$

Notice that in general, if it is $a_{h}+a_{f}>1$ then the premises in the question are false for this level, and it is nonsense to look for an answer in this level. We assume here this is not the case. On the other hand, if $b_{h}+b_{f}>1$ we can take just 1 as the upper bound of the interval since we know that no more than $100 \%$ of elements can verify $A \vee B$. Then

$$
\left.\left[a_{C}, b_{C}\right]=\left[\left(1-b_{A \vee B}\right) \times a_{m},\left(1-a_{A \vee B}\right]\right) \times b_{m}\right]
$$

Hence

$$
\left[a_{\neg D}, b_{\neg D}\right]=\left[a_{A \vee B}+a_{C}, \min \left\{1, b_{A \vee B}+b_{C}\right\}\right]
$$

assuming again that $a_{A \vee B}+a_{C} \leq 1$ since otherwise the premises are false in this level, and again restricting the maximum to 1 . Finally,

$$
\left[a_{D}, b_{D}\right]=\left[1-b_{\neg D}, 1-a_{\neg D}\right]
$$


In order to calculate the RL answering the question, we proceed as follows: first, we applied the procedure just described in level 1 (the less restrictive). If the premises of the question are false for this level, then the premises are false and we cannot give an answer (notice that the definition of the quantifiers are considered part of the premises). Otherwise, we obtain an interval in $[0,1]$. Then, the procedure is repeated in every level from higher to lower values of $\alpha$ until either we reach to the last level, or the premises are false for some level. In the latter case, we consider that the interval obtained in the last level in which the premises where valid is the interval for the rest of the levels with lower $\alpha$.

Notice that the intervals may not be nested, and hence the result may not be a fuzzy quantifier. However, we can provide a fuzzy quantifier as the single-point coverage function, as explained in section II. It is assumed however that, in case there are further operations or questions about the amount of elements satisfying $D$, it is the RL of this information which have to be used, the fuzzy quantifier being just information for the human user.

In order to illustrate our approach, suppose we are considering levels $\Lambda=\{1,0.8,0.6,0.4,0.2\}$ and we have the following quantifiers:

$$
\begin{gathered}
Q_{h}(\beta)= \begin{cases}0 & \beta \leq 0.4 \\
10 \times(\beta-0.4) & 0.4 \leq \beta \leq 0.5 \\
10 \times(0.6-\beta) & 0.4 \leq \beta \leq 0.6 \\
0 & 0.6 \leq \beta\end{cases} \\
Q_{f}(\beta)= \begin{cases}1 & \beta \leq 0.1 \\
5 \times(0.3-\beta) & 0.1 \leq \beta \leq 0.3 \\
0 & 0.3 \leq \beta\end{cases} \\
Q_{m}(\beta)= \begin{cases}0 & \beta \leq 0.7 \\
10 \times(\beta-0.7) & 0.7 \leq \beta \leq 0.8 \\
1 & 0.8 \leq \beta\end{cases}
\end{gathered}
$$

Table VII shows the computations. In this particular case, the premises were not false for any level. Also, the result is a collection of nested intervals, so it is directly a fuzzy set that can be approximated by the following quantifier $Q_{D}$ :

$$
Q_{f}(\beta)= \begin{cases}1 & \beta \leq 0.18 \\ 1 / 0.35 \times(0.53-\beta) & 0.18 \leq \beta \leq 0.53 \\ 0 & 0.53 \leq \beta\end{cases}
$$

\section{COnClusions}

The representation and operations by levels independently provides a way to represent fuzziness keeping the Boolean properties and having at the same time elements in both $A$ and $\neg A$ for any fuzzy concept $A$ which is not crisp. This Boolean structure allows to obtain more intuitive results when working with fuzzy quantification. In addition, the problem of fuzzy quantification can be transformed into a collection of crisp versions that can be solved using the existing solutions for the crisp case. We have provided an example in solving a typical question in Computing with Words. We will continue the line sketched here in future work.

\section{ACKNOWLEDGMENTS}

The research reported in this paper was partially supported by the Andalusian Government (Junta de Andalucía) under project P07-TIC03175 and from the former Spanish Ministry for Science and Innovation by the project grants TIN200914538-C02-01 and TIN2009-08296.

\section{REFERENCES}

[1] L. Zadeh, "A new direction in AI. Toward a computational theory of perceptions," AI Magazine, vol. 22, no. 1, pp. 73-84, 2001.

[2] L. A. Zadeh, "A computational approach to fuzzy quantifiers in natural languages," Computing and Mathematics with Applications, vol. 9, no. 1, pp. $149-184,1983$.

[3] D. Dubois and H. Prade, "Fuzzy cardinality and the modeling of imprecise quantification," Fuzzy Sets and Systems, vol. 16, pp. 199-230, 1985.

[4] R. Yager, "On ordered weighted averaging aggregation operators in multicriteria decisionmaking," IEEE Transactions on System, Man and Cybernetics, vol. 18, no. 1, pp. 183-190, 1988.

[5] M. Delgado, D. Sánchez, and M. Vila, "Fuzzy cardinality based evaluation of quantified sentences," International Journal of Approximate Reasoning, vol. 23, pp. 23-66, 2000.

[6] F. Díaz-Hermida, D. E. Losada, A. Bugarín, and S. Barro, "A probabilistic quantifier fuzzification mechanism: The model and its evaluation for information retrieval." IEEE Transactions on Fuzzy Systems, vol. 13 , no. 5 , pp. $688-700,2005$.

[7] I. Glöckner, Fuzzy Quantifiers: A Computational Theory. Springer, 2006.

[8] M. Ying, "Linguistic quantifiers modeled by sugeno integrals," Artificial Intelligence, vol. 170, pp. 581-606, 2006.

[9] L. Cui and Y. Li, "Linguistic quantifiers based on choquet integrals," Int. Journal of Approximate Reasoning, vol. 48, pp. 559-582, 2008.

[10] L. Lietard and D. Rocacher, "Evaluation of quantified statements using gradual numbers," in Handbook of Research on Fuzzy Information Processing in Databases Vol., J. Galindo, Ed. Hershey, PA, USA, 2008, pp. 246-269.

[11] D. Sánchez, M. Delgado, and M. Vila, "Fuzzy quantification using restriction levels," in Proc. WILF 2009., ser. LNCS. Springer, 2009, vol. 5571, pp. 28-35.

[12] D. Dubois and H. Prade, "New results about properties and semantics of fuzzy set- theoretic operators," in Fuzzy Sets: Theory and Applications to Policy Analysis and Information Systems, P. Wang and S. Chang, Eds. Plenum Publ., 1980, pp. 59-75.

[13] — , "An introduction to possibility and fuzzy logics," in Non-Standard Logics for Automated Reasoning, P. S. et.al., Ed. Academic Press, 1988, pp. $742-755$.

[14] D. Sánchez, M. Delgado, M. Vila, and J. Chamorro-Martínez, "On a nonnested level-based representation of fuzziness," Fuzzy Sets and Systems, vol. 192, pp. 159-175, 2012.

[15] D. Dubois and H. Prade, "Fuzzy elements in a fuzzy set," in Proc. IFSA'05, 2005, pp. 55-60.

[16] _ - "Gradual elements in a fuzzy set," Soft Computing, vol. 12, pp. $165-175,2008$.

[17] — "The three semantics of fuzzy sets," Fuzzy Sets and Systems, vol. 90, pp. 141-150, 1997.

[18] G. Shafer, A Mathematical Theory of Evidence. Princeton University Press, 1976.

[19] H. Nguyen, "Some mathematical tools for linguistic probabilities," Fuzzy Sets and Systems, vol. 2, pp. 53-65, 1979.

[20] —, An introduction to random sets. Chapman \& Hall/CRC, 2006.

[21] A. Pradera, E. Trillas, S. Guadarrama, and E. Renedo, "On fuzzy set theories," in Fuzzy Logic, P. Wang and D. Ruan, Eds. Springer, 2007, vol. 215, pp. 15-47. 